\title{
The Luminescent Property of Magnetic Polymers and Its Influencing Factors
}

\author{
Zeren $\mathrm{Li}^{1, *}$ \\ ${ }^{1}$ School of Material Science and Engineering, Tianjin University, Tianjin, China
}

\begin{abstract}
In this paper, the generation mechanism, influencing factors and application of the luminescent properties of magnetic polymers are reviewed. In practical applications, magnetic polymers exhibit a variety of unique luminescent properties, including photoluminescence, electroluminescence and thermoluminescence. There are many factors affecting the luminescent properties of different kinds of magnetic polymers, including the types of magnetic centers, the properties of metal ions, the charge transfer between metal ions and ligands, and the influence of functional groups on the main and side chains of the polymers. This paper explains the luminescent properties of magnetic polymers from the aspects of generation mechanism and influencing factors.
\end{abstract}

\section{Introduction}

In recent years, with the continuous development of polymer research, a variety of polymers with unique properties have been developed and received wide applications. Magnetic polymer is one type of them. Magnetic polymer is a kind of macromolecular polymer which usually shows ferromagnetism or paramagnetism. Its structure is usually composed of polymer positive ions and inorganic negative ions. Among them, organic positive ions usually include imidazole, pyridine nitrogen ions, alkyl amines and alkyl phosphines, while negative ions usually include halides (such as [FeCl4]), mineral anions and polyatomic inorganic substances [1]. According to the different composition of anions and cations, magnetic polymers can be divided into two types. One type is the magnetic polymer prepared by coating the polymer film on the magnetic particles (See Figure 1 as an example). The magnetic particles can generally be made of magnetic materials such as $\mathrm{Fe} 3 \mathrm{O} 4$. The characteristic of this magnetic polymer is that the polymer and magnetic particles are relatively independent, and the physical and chemical properties of one part have little influence on the other part. For example, the magnetic polymer microcapsule prepared by using polymethylacrylic acid and $\mathrm{Fe} 3 \mathrm{O} 4$ was used to make drug carriers for targeted therapy [2]. The other is the magnetic polymer containing metal ions or complexes, whose magnetism is mainly realized by magnetic coupling between the transition metal in anion complexes. Using these anions and cations to form complexes, the magnetic polymer can maintain its magnetic characteristics, and at the same time, obtain the polymer's characteristics of easy structure adjustment and chemical modification. Magnetic polymer is characterized by its simple preparation and mild reaction conditions, and its magnetism is convenient for secondary separation, purification and recycling.

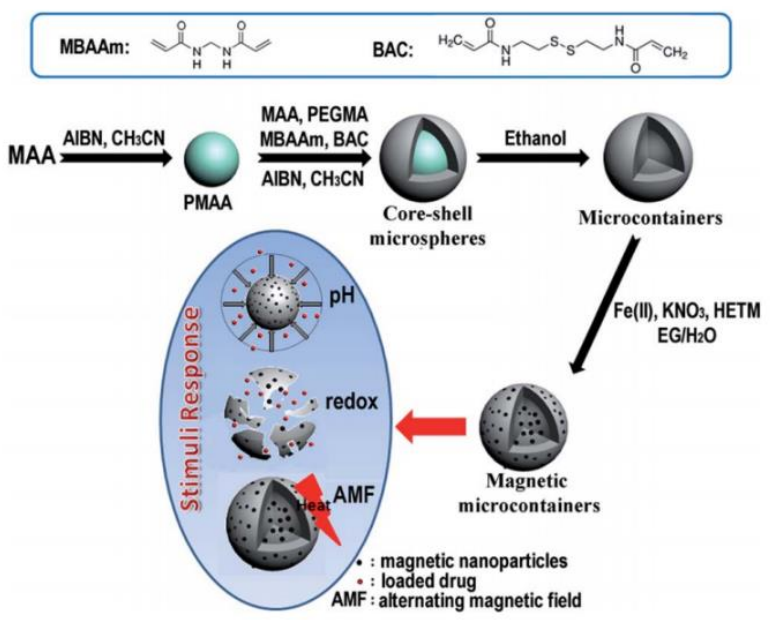

Figure 1 Magnetic polymer micro-container with magnetic nanoparticles as magnetic center

The luminescent properties of magnetic polymers have wide applictions. In the field of organic catalysis, the catalyst prepared by using the fluorescence property of magnetic polymer makes it more convenient to detect the amount and intensity of the catalyst being used. For example, in the experiment of catalyzing the degradation of polylactic acid, the efficiency and consumption of the catalyst based on 1-Butyl-3-methylimidazole and [FeCl4] magnetic polymer can be effectively detected by optical, thermal, and other conveneient means if fluorescent groups can be introduced into the cationic part of the polymer [3]. Also, during the preparation of dysprosium containing magnetic polymer, the luminescence of dysprosium can be observed to verify whether dysprosium ion is successfully reacted into the magnetic polymer [4]. In the aspect of organic extraction, the products can be 
separated well depending on the luminescent properties of magnetic polymers. In biological and medical applications, the photochromic and thermochromic properties of magnetic polymers can also be used to prepare biosensors for transmitting biological signals.

\section{Fluorescence generation principle of magnetic polymers}

The main mechanism of the magnetic polymer's luminescent property is that when the material's surface is irradiated by visible light or ultraviolet light, the energy of photons is transferred to the electrons inside the polymer. After receiving the energy, the electrons will jump out of their energy level and release energy at the same time. This energy is released in the form of photons, which forms the photoluminescence characteristic of materials. Due to the influence of metal ligands and functional groups of different types, the conditions under which they are excited by photons are different, which means the luminescence phenomenon of magnetic polymers response to different wavelengths under the irradiation of ultraviolet and visible light.

The factors that may affect the fluorescence reaction and spectral analysis of magnetic polymers include the following aspects. One is the internal relaxation process of polymer. In previous studies [5], it was found that there are three relaxation processes in polyethylene molecular chain, including $\alpha$ relaxation, $\beta$ relaxation and $\gamma$ relaxation. Among them, $\gamma$ relaxation is caused by the start or stop of the movement of small polymer segments in the amorphous region. The observation of these three relaxation processes can be determined by the fluorescence changes in substitution of anthracene doped in the polymer or the anthracene group covalently attached to the polymer chain. In addition to the influence of the relaxation, the fluorescence properties will also be affected by temperature. For example, the fluorescence band of pyran group will have redshift with the increase of temperature.

The optical properties of pyrene groups attached to the surface of polyethylene are characterized. The determination results of $7.0 \mathrm{MeV}$ radiation bombardment shows that the split $0-0$ bands are distributed to two different pyrene groups on the surface of polyethylene, which indicates that different pyrene attached polymers has different absorption and emission properties. At the same time, only one spectrum is observed in the emission spectrum band of the samples irradiated by eV-range photons, which indicates that there is only one corresponding pyrene group on each polyethylene chain. With these findings, it can be concluded that when the absorption and emission spectra are independent of wavelength and have the same shape, position and time attenuation characteristics, the photoluminescence properties of this polymer are relatively poor. In addition, the emission spectrum intensity of pyrene group will decrease with the increase of temperature, and its intensity cannot be recovered after cooling, which may be due to the decomposition of pyrene group or the change of physical properties at high temperature.

\section{Influencing factors of magnetic polymer luminescent properties}

In different types of magnetic polymers, their luminescent properties are affected by many factors, which can be roughly divided into the influence of metal ions and complexes, the influence of main chain and side chain functional groups, the influence of the structure between polymer chains, and the influence of temperature and current, etc. This paper will focus on the influence of metal ions and complexes as well as the influence of main chain and side chain functional groups.

\subsection{Influence of metal ions and complexes on luminescent properties}

In magnetic polymers, it is common to use metal ions and complexes to make polymers achieve luminescent properties. The common metal elements used include Fe, $\mathrm{Al}, \mathrm{Cu}, \mathrm{Co}$ and so on. Different metals have different effects on polymers. For instance, the luminescent properties of organometallic polymers can be divided into the following categories, including center luminescence, Metal-to-Ligand Charge Transfer (MLCT), Ligand-toMetal Charge Transfer (LMCT), Ligand-to-Ligand Charge Transfer (LLCT), ligand center luminescence and Metal-to-Metal Charge Transfer [6]. Among these properties, both metal center luminescence and ligand center luminescence are affected by the properties of the metal or ligand itself, that is, the unique fluorescence characteristics of luminescent groups, and have nothing to do with the charge transfer between molecules and atoms. The influence of MLCT, LMCT and LLCT on the luminescent properties mainly shows that charge transfer changes the fluorescence group structure in the magnetic polymer, and then changes the emission spectrum energy under light or heating conditions. For example, in the magnetic polymers containing $\mathrm{Zn}$ and $\mathrm{Cd}$, the molecular structure of $\mathrm{Zn}$ (II) and $\mathrm{Cd}(\mathrm{II})$ has the function of double permeation, the main effect of which on the luminescent properties of the polymer is that the metal increases the substituent carboxylate in BTC, and increases the HOMO energy and reduces the LUMO energy to change its luminescent property. Another influence of the metal element or metal ion on the luminescent property of the magnetic polymer is the metal connector. The composition of this kind of metal ions and complexes, such as $\mathrm{Cu}, \mathrm{Zn}$ and $\mathrm{Mn}$, will lead to the increase of connector hardness and the decrease of non-radiation attenuation rate. In this case, the increase of fluorescence lifetime and quantum yield can usually be observed, and the fluorescence emission intensity of the polymer is enhanced. In MLCT, a common influencing factor is to transfer the charge from the metal center to the connector to generate excitation effect. The experiments show that the addition of $\mathrm{Cu}$ into the polymer and its complex ions may make the luminescence properties invalid and show different luminous intensity.

In addition, the presence of spin crossover can also influence the luminescent properties of magnetic polymers. For example, he magnetic polymer with [Tf2N] 
as coordination ions can achieve the conversion between high spin and low spin and further the thermochromic effects through the change of the length of iron-oxygen bond. Moreover, as temperature changes, the magnetic properties of magnetic polymers will also change accordingly [7].

\subsection{Influence of functional groups on polymer monomers}

In magnetic polymers, the molecular groups and functional groups on the monomers also affect the luminescent properties. For example, some polymer monomer contains the pyrene group. Pyrene's supramolecular effect, also known as p-p stacking, affects the space between the polymer molecular chains, as well presents different optical properties under the influence of different functional groups [8]. For example, after adding Bpin groups to both ends of pyrene and making single-end modification and double-end modification, it showed obvious differences in the position of the UV fluorescence points on the column plate. Such changes in fluorescence properties make pyrene polymers a good indicator to reflect the degree and stage of the reaction in the laboratory. With such property, one can get timely in-situ result when the reaction enters certain stages and have a more efficient and convenient processing in the later on edulcoration and purification procedures. Also, in the copolymers of polyvinyl imidazole and polypyridine methyl methacrylate, the pyrene group can bring the polymer fluorescence property, without affecting the superparamagnetism of the materials, thus can be used in the fields of biological transfer and targeted therapy. In addition, polymer monomers such as fluorene and naphthalene exhibit different fluorescence phenomena under UV fluorescence. Fei Huang et al. prepared a kind of polymer monomer which substituted fluorene with bromine, and copolymerized with 2,7-bis(4,4,5,5tetramethyl-1,3,2-dioxaborolan-2-yl)-9,9-dioctylfluorene and 1,4-phenyldiboronic acid respectively, and modified the quaternary ammonium salt ions on the side chain to obtain four polymers of P1-P4 [9], as illustrated in Figure 2 [10]. After testing the electroluminescence performance of the four polymers, it showed that the spectra of the two polymers (Table 1) modified by quaternary ammonium salt were more similar to the typical emission spectra of polyfluorene compounds, in which EL was coincided with PL, and the other peak was located at $490 \mathrm{~nm}$, which belonged to excimer emission. Moreover, the peak wavelengths of the two polymers were shorter than those of the corresponding polymers without bromine ion. This indicates that the quaternary ammonium salt ions in the side chain can also affect the electroluminescence performance of the whole polymer.

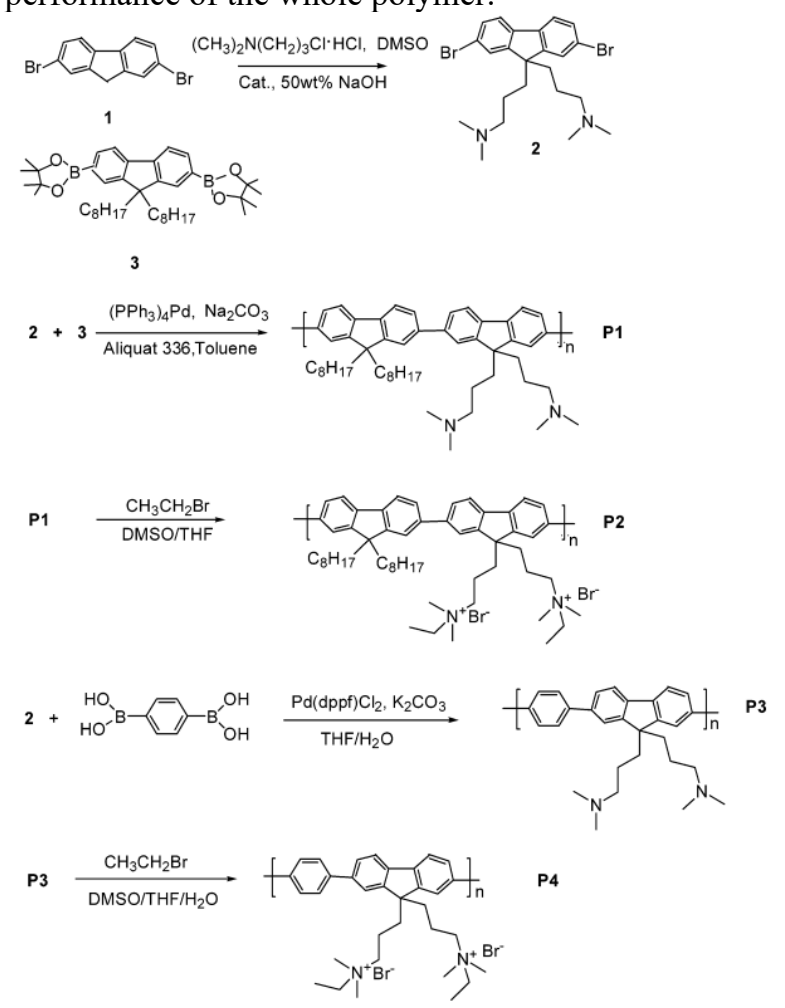

Figure 2 Synthesis of several fluorene-containing polymers

Table 1 UV-Vis Absorption, Electrochemical, and Photoluminescence Properties and Electroluminescence of polymers.

\begin{tabular}{|c|c|c|c|c|c|c|c|c|c|c|}
\hline \multirow[b]{2}{*}{ polymers } & \multirow[b]{2}{*}{$\lambda \operatorname{absmax}(\mathrm{nm})$} & \multirow{2}{*}{$\begin{array}{c}\text { optical } \\
\text { band } \\
\text { gap }^{\mathrm{a}} \\
(\mathrm{eV})\end{array}$} & \multirow[b]{2}{*}{$\operatorname{Eox}(\mathrm{V})$} & \multirow[b]{2}{*}{$\operatorname{Ered}(\mathrm{V})$} & \multirow[b]{2}{*}{$\begin{array}{c}\text { HOMO } \\
(\mathrm{eV})\end{array}$} & \multirow[b]{2}{*}{$\begin{array}{l}\text { LUMO } \\
(\mathrm{eV})\end{array}$} & \multirow[b]{2}{*}{$\operatorname{Egap}(\mathrm{eV})$} & \multicolumn{2}{|c|}{ photoluminescence } & \multirow[b]{2}{*}{$\begin{array}{l}\text { electroluminescence } \\
\lambda \operatorname{ELmax}(\mathrm{nm})\end{array}$} \\
\hline & & & & & & & & $\lambda \operatorname{PLmax}(\mathrm{nm})$ & QPLmax (\%) & \\
\hline P1 & 390 & 2.91 & 1.21 & -2.26 & -5.61 & -2.14 & 3.47 & 425,449 & 37.3 & 515 \\
\hline $\mathbf{P 2}$ & 400 & 2.85 & 1.25 & -2.22 & -5.65 & -2.18 & 3.47 & 431,455 & 4.7 & 433,492 \\
\hline P3 & 382 & 2.96 & 1.3 & -2.28 & -5.7 & -2.12 & 3.58 & 420,444 & 18.5 & 535 \\
\hline P4 & 388 & 2.92 & 1.33 & -2.29 & -5.73 & -2.11 & 3.62 & 425,447 & 8.3 & 442,497 \\
\hline
\end{tabular}

\section{Conclusion}

To sum up, this paper summarizes the mechanism of the luminescent properties of magnetic polymers, as well as the major factors that may affect it. In magnetic polymers, the type of magnetic center, the type of metal ions in the 
magnetic center, the charge transfer between metal ions and ligands, and the properties of functional groups on the polymer chain are important factors affecting the final luminescent properties. With the deepening of the research on magnetic polymers, more aspects and in-depth influencing factors will be more systematically studied, and the luminescent properties of magnetic polymers therefore can find more application in the field.

\section{References}

1. Jiayin Yuan, Markus Antonietti. Poly (ionic liquid) s: Polymers expanding classical property profiles. Polymer, 2011, 52, 1469-1482.

2. Panayiotis Bilalis, Alexandros Chatzipavlidis, LetoAikaterini Tziveleka. Nanodesigned magnetic polymer containers for dual stimuli actuated drug controlled release and magnetic hyperthermia mediation. J. Mater. Chem., 2012, 22, 13451.

3. Huiqing Liu, Ruiyang Zhao, Xiuyan Song, et al. Lewis acidic ionic liquid [Bmim] FeCl4 as a high efficient catalyst for methanolysis of poly (lactic acid) [J]. Catal. Lett., 2017, 147, 2298-2305.

4. Bert Mallick, Benjamin Balke, Claudia Felser, et al. Dysprosium room-temperature ionic liquids with strong luminescence and response to magnetic fields[J]. Angew. Chem. Int. Ed., 2008, 47, 1-5.

5. Gerald O. Brown, Teresa D. Z. Atvars, Noel A. Guardala, et al. Temperature-dependent fluorescence from pyrenyl-modified polyethylene films. A comparison of attachment methods and morphology changes by irradiation with ev-range photons and bombardment with mev-range positive ions. J. Polym. Sci. B, 2004, 42, 2957-2970.

6. M. D. Allendorf, C. A. Bauer, R. K. Bhaktaa and R. J. T. Houk. Luminescent metal-organic frameworks. Chem. Soc. Rev., 2009, 38, 1330-1352.

7. Megumi Okuhata, Yusuke Funasako, Kazuyuki Takahashi and Tomoyuki Mochida. A spincrossover ionic liquid from the cationic iron (III) Schiff base complex. Chem. Commun., 2013, 49, 7662-7664.

8. Andrew G. Crawford, Zhiqiang Liu, Ibraheem A. I. Mkhalid, et al. Synthesis of 2- and 2,7-functionalized pyrene derivatives: An application of selective $\mathrm{C}-\mathrm{H}$ borylation. Chem. Eur. J. 2012, 18, 5022-5035.

9. Fei Huang, Hongbin Wu, Deli Wang, Wei Yang, and Yong Cao. Novel electroluminescent conjugated polyelectrolytes based on polyfluorene. Chem. Mater., 2004, 16, 708-716.

10. Fei Huang, Hongbin Wu, Deli Wang, Wei Yang, and Yong Cao. Novel electroluminescent conjugated polyelectrolytes based on polyfluorene. Chem. Mater., 2004, 16, 708-716. 